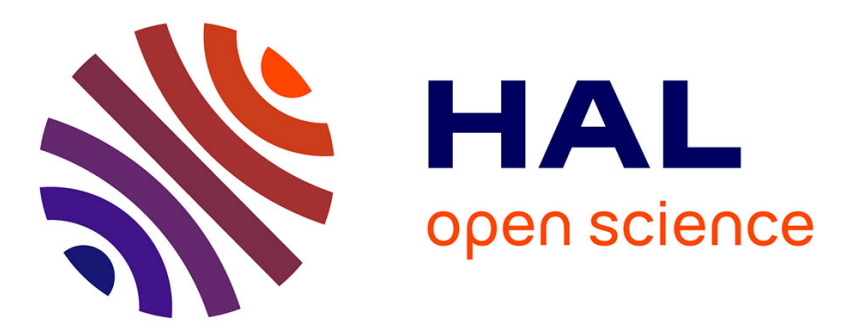

\title{
Axisymmetric deformations of circular rings made of linear and Neo-Hookean materials under internal and external pressure: A benchmark for finite element codes
} Ivan Breslavsky, Marco Amabili, Mathias Legrand, Farbod Alijani

\section{- To cite this version:}

Ivan Breslavsky, Marco Amabili, Mathias Legrand, Farbod Alijani. Axisymmetric deformations of circular rings made of linear and Neo-Hookean materials under internal and external pressure: A benchmark for finite element codes. International Journal of Non-Linear Mechanics, 2016, 84, pp.3945. 10.1016/j.ijnonlinmec.2016.04.011 . hal-01339533

\section{HAL Id: hal-01339533 https://hal.science/hal-01339533}

Submitted on 29 Jun 2016

HAL is a multi-disciplinary open access archive for the deposit and dissemination of scientific research documents, whether they are published or not. The documents may come from teaching and research institutions in France or abroad, or from public or private research centers.
L'archive ouverte pluridisciplinaire HAL, est destinée au dépôt et à la diffusion de documents scientifiques de niveau recherche, publiés ou non, émanant des établissements d'enseignement et de recherche français ou étrangers, des laboratoires publics ou privés.

\section{(c)(1)}

Distributed under a Creative Commons Attribution| 4.0 International License 


\title{
Axisymmetric deformations of circular rings made of linear and Neo-Hookean materials under internal and external pressure: A benchmark for finite element codes
}

\author{
Ivan D. Breslavsky ${ }^{a}$, Marco Amabili ${ }^{\mathrm{a}, \mathrm{b}, *}$, Mathias Legrand $^{\mathrm{a}}$, Farbod Alijani ${ }^{\mathrm{c}}$ \\ a Department of Mechanical Engineering, McGill University, 817 Sherbrooke Street West, Montréal, Québec, Canada H3A 0C3 \\ ${ }^{\mathrm{b}}$ Department of Industrial Engineering, University of Parma, Parco Area delle Scienze 181/A, Parma, Italy \\ ${ }^{\mathrm{c}}$ Department of Precision and Microsystems Engineering, Delft University of Technology, Mekelweg 2, 2628 CD Delft, The Netherlands
}

The axisymmetric deformations of thick circular rings are investigated. Four materials are explored: linear material, incompressible Neo-Hookean material and Ogden's and Bower's forms of compressible Neo-Hookean material. Radial distributed forces and a displacement-dependent pressure are the ex-ternal loads. This problem is relatively simple and allows analytical, or semi-analytical, solution; therefore it has been chosen as a benchmark to test commercial finite element software for various material laws at large strains. The solutions obtained with commercial finite element software are almost identical to the present semi-analytical ones, except for the linear material, for which commercial finite element programs give incorrect results.

\section{Introduction}

The study of circular rings, as well as of associated problems of infinite circular cylindrical shells under pressure, has a long history. In the context of infinitely small deformations and linear elasticity, a solution was brought in the middle of the XIXth century by Lamé [1]. Complementary references to early studies of the problem are given in the book by Love [2]. A hundred years after Lamé's solution, formulations incorporating nonlinear materials were proposed by Lurie [3]. In this book, axially symmetric (i.e. being circular, angle-independent and with the same radius along the axis, even if the radius can obviously change due to loads with respect to the unloaded configuration) deformations are assumed, and the formula describing the internal radius of long cylinders made of semi-linear materials, subjected to an external pressure, is obtained. The closed-form stress distribution within a cylinder of incompressible, Mooney-Rivlin hyperelastic material is also provided.

Thick-walled hollow cylinders under pressure were examined in a series of papers by Ogden and co-authors [4-7], where a fully threedimensional finite element approach is considered to build numerical solutions. In [5] the hollow cylinders and rings made of Ogden's hyperelastic incompressible material, subjected to a combination of axial loading and external pressure, are investigated. The ends of the cylinder are constrained, so that no displacements are allowed. It is found that the mode with two circumferential waves becomes the sole dominant buckling mode as the length of the hollow cylinder increases. For very short and sufficiently thick cylinders, transition from lower to higher (i.e. with more circumferential waves) modes occurs in the considered range of axial compression. There is an extensive literature on the buckling and post-buckling of rings and circular cylindrical shells under pressure; however, in the present review, only studies on axially symmetric deformations (i.e. the ring keeps a circular cross-section) are targeted. In [6] the axisymmetric deformations of hollow cylinders under external pressure are considered. The cylinders are made of incompressible Neo-Hookean material. Both long and short circular cylindrical shells with zero displacements at the ends are considered. The authors pay special attention to the radially-symmetric deformation and it is found that, for moderate deflections, the solution with prescribed axial symmetry is close to the actual solution. Also, a comparison with the solution to the underlying linearized formulation is proposed. As expected, this solution compares well with the fully nonlinear solution for very small deflections only. Unfortunately, the analysis does not consider the separate effect of physical and geometrical nonlinearities, but it is observed that the physical nonlinearity dominates in shorter and thicker hollow cylinders, while geometrical nonlinearity has a leading effect for longer and thinner cylinders.

The deformations of thick circular cylindrical shells under external and internal pressure are investigated in [7]. Again, zero 
displacements are assumed at the shell ends. The paper specifically explores the buckling, but the range of external pressure magnitudes for which the shell remains circular, are also provided. In general, the authors come to the conclusion that, for thickwalled short cylinders under either internal or external pressure, the radially symmetric solution provides a very good approximation of the actual solution.

In the paper by Erbay and Demiray [8], radial and axial deformations of Neo-Hookean hollow cylinders under extension and inflation are studied. The stress distribution in the special case of deformation preserving circular cylindrical shape is discussed.

In [9], a nonlinear stability analysis for deformations of incompressible hyperelastic solids is presented. The conditions driving the existence and uniqueness of cylindrical deformations for hollow cylinders under internal pressure are derived.

In $[10,11]$, the pre-buckling behavior of hollow cylinders under external pressure is discussed. It is found that thick-walled cylinders preserve a symmetric shape for external loads much higher than for thin-walled cylinders.

In the present study, we investigate the static, axially symmetric deformations of a free-end ring under internal and external pressures. A few types of material are studied: a typically linear material that obeys Hooke's law, an incompressible Neo-Hookean material, and two types of compressible Neo-Hookean material. A simplified geometrically nonlinear shell theory is considered to describe the strain-deflection relationships. In the case under consideration, the deformation is governed by a single ordinary differential equation. Two loads are considered: the first one is a uniformly distributed radial force (displacement independent pressure), while the second one is a displacement-dependent pressure (actual pressure).

The problem under study is relatively simple and allows analytical or semi-analytical solution. Therefore, it has been chosen as a benchmark to test commercial finite element software for different material laws at large strains. Numerical results are compared with the predictions of the commercial finite element programs ANSYS and ABAQUS. The solutions obtained with these programs are almost identical to the present semi-analytical ones, except for the linear material, for which commercial finite element programs give incorrect results. Instead, a successful comparison with results obtained by a non-commercial finite element program developed by the group of Professor Reddy [18] is shown for the case of linearly elastic material.

\section{Strain tensors}

Different materials are implemented in this study: a physically linear elastic material and three types of Neo-Hookean hyperelastic materials. These two families of materials are defined in terms of their strain energy densities (SED). The SED for linear elastic material is expressed in terms of the components of the Green-Lagrange strain tensor E, which in cylindrical coordinates $z, \theta, x$ (see Fig. 1) takes the following form:

$\mathbf{E}=\left(\begin{array}{ccc}\varepsilon_{x} & \varepsilon_{x \theta} / 2 & 0 \\ \varepsilon_{x \theta} / 2 & \varepsilon_{\theta} & 0 \\ 0 & 0 & \varepsilon_{z}\end{array}\right)$.

Here the transformation $r=R+z$ has been used, where $z$ has the same direction of the radial coordinate $r$ but it is measured from the middle surface of the ring instead of the ring longitudinal axis $x$.

In case of axially symmetric deformations of a ring under uniform pressure, the shear deformation vanishes $\left(\varepsilon_{x \theta}=0\right)$ and the tensor $\mathbf{E}$ is thus diagonal.

The SED, for all variants of Neo-Hookean materials, is given as a function of the invariants of the Cauchy-Green deformation tensor.

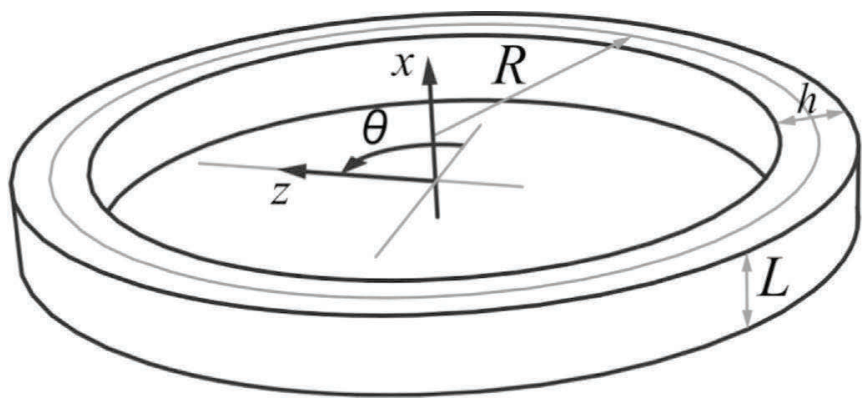

Fig. 1. Sketch of the ring and cylindrical coordinate system.

Since we approach the problem in term of displacements (see for example $[12,13])$, it is useful to express the right Cauchy-Green deformation tensor $\mathbf{C}$ in terms of $\mathbf{E}$ :

$\mathbf{C}=2 \mathbf{E}+\mathbf{I}=\left(\begin{array}{ccc}2 \varepsilon_{x}+1 & 0 & 0 \\ 0 & 2 \varepsilon_{\theta}+1 & 0 \\ 0 & 0 & 2 \varepsilon_{z}+1\end{array}\right)$

On the other hand, $\mathbf{C}$ can be represented as matrix with squared principal stretches as diagonal elements:

$\mathbf{C}=\left(\begin{array}{ccc}\lambda_{x}^{2} & 0 & 0 \\ 0 & \lambda_{\theta}^{2} & 0 \\ 0 & 0 & \lambda_{z}^{2}\end{array}\right)$.

The principal stretches, as functions of the Green-Lagrange strain tensor components, can be easily obtained by identifying the corresponding components of $\mathbf{C}$ in (1) and (2). Finally, the invariants of the tensor $\mathbf{C}$ involved in the calculation of SEDs are defined:

$I_{1}=\operatorname{Tr}(\mathbf{C})=2\left(\varepsilon_{x}+\varepsilon_{\theta}+\varepsilon_{z}\right)+3=\lambda_{x}^{2}+\lambda_{\theta}^{2}+\lambda_{z}^{2}$,

$J=\sqrt{|\mathbf{C}|}=\sqrt{\left(2 \varepsilon_{X}+1\right)\left(2 \varepsilon_{\theta}+1\right)\left(2 \varepsilon_{z}+1\right)}=\lambda_{x} \lambda_{\theta} \lambda_{z}$.

\section{Strain-displacement relationships and strain energy densities}

\subsection{Circumferential strain}

Consider a ring of radius $R$, thickness $h$ and length $L$ with free ends (see Fig. 1). In this study we assume that $L=h$. The ring is modeled as a very short circular cylindrical shell and associated derivations stem from the theory of thin shells. The expression for the circumferential strain is [14]

$$
\begin{aligned}
\epsilon_{\theta}= & \frac{1}{R} \frac{\partial v}{\partial \theta}+\frac{w}{R}+\frac{1}{2}\left(\left(\frac{\partial u}{\partial \theta}\right)^{2}\right)+\left(\frac{1}{R} \frac{\partial v}{\partial \theta}+\frac{w}{R}\right)^{2}\left(+\left(\frac{1}{R} \frac{\partial w}{\partial \theta}-\frac{v}{R}\right)^{2}\right) \\
& +z\left(\frac{1}{R} \frac{\partial u}{\partial x}+\frac{1}{R^{2}} \frac{\partial v}{\partial \theta}-\frac{1}{R^{2}} \frac{\partial^{2} w}{\partial \theta^{2}}\right),
\end{aligned}
$$

where $u(z, \theta, x), v(z, \theta, x), w(z, \theta, x)$ are the displacements of a generic point belonging to the middle surface in the directions of the cylindrical coordinate system $z, \theta, x$, respectively.

The ring and the load are both axisymmetric. Accordingly, it is assumed that the ring remains circular after the deformation with new radius $R+w$. Note that the present work investigates axially symmetric deformations only and buckling is ignored. Due to the prescribed symmetry, the displacements do not depend on the angular coordinate $\theta$, and the displacement in the circumferential direction $v(z, \theta, x)$ is assumed to vanish. Finally, it seems reasonable 
to assume that the shortness of the shell is such that the term $\frac{\partial u}{\partial x}$ can be neglected, i.e. $u$ is assumed to be constant along the ring length since the ring is really short. Accordingly, the circumferential strain takes the following simplified expression:

$\varepsilon_{\theta}=\frac{w}{R}+\frac{w^{2}}{2 R^{2}}$.

\subsection{Non-vanishing strains in case of linear material}

For a linear material, the axial strain is expressed by enforcing a zero axial stress that is [14]

$\sigma_{X}=\frac{E}{1-v^{2}}\left(\varepsilon_{X}+v \varepsilon_{\theta}\right)=0$,

where $E$ and $v$ stand for Young's modulus and Poisson's ratio of the material, respectively. In Eq. (6), plain stress has been assumed, i.e. $\sigma_{z}=\tau_{x z}=\tau_{\theta z}=0$. The assumption $\sigma_{x}=0$ is due to the shortness of the ring in axial direction and the fact that the ring is neither subjected to kinematic constraints nor axial load. This implies

$\varepsilon_{x}=-v \varepsilon_{\theta}$.

The corresponding strain energy density for the physically linear material is [12-14]

$W_{L}=\frac{1}{2}\left(\epsilon_{\chi} \sigma_{x}+\epsilon_{\theta} \sigma_{\theta}\right)=\frac{1}{2} \epsilon_{\theta} \sigma_{\theta}$.

The expression for the axial stress is [14]

$\sigma_{\theta}=\frac{E}{1-v^{2}}\left(\varepsilon_{\theta}+v \varepsilon_{X}\right)=\frac{E}{1-v^{2}}\left(1-v^{2}\right) \varepsilon_{\theta}=E \varepsilon_{\theta}$,

Inserting the above equality in (7) leads to:

$W_{L}=\frac{1}{2} \varepsilon_{\theta} \sigma_{\theta}=\frac{E}{2} \varepsilon_{\theta}^{2}$

The total elastic potential energy is retrieved by integrating the strain energy density over the domain defining the ring that is:

$$
\begin{aligned}
U_{L} & =\frac{E}{2} \int_{0}^{L} \int_{0}^{2 \pi} \int_{-\frac{h}{2}}^{\frac{h}{2}} \epsilon_{\theta}^{2} R\left(1+\frac{z}{R}\right) d x d \theta d z \\
& =\frac{E}{2} \int_{0}^{L} \int_{0}^{2 \pi} \int_{-h / 2}^{h / 2}\left(\frac{w^{2}}{R^{2}}+\frac{w^{3}}{R^{3}}+\frac{w^{4}}{4 R^{4}}\right) R\left(1+\frac{z}{R}\right) d x d \theta d z \\
& =E R L \pi h\left(\frac{w^{2}}{R^{2}}+\frac{w^{3}}{R^{3}}+\frac{w^{4}}{4 R^{4}}\right)=\frac{E L \pi h}{R}\left(w^{2}+\frac{w^{3}}{R}+\frac{w^{4}}{4 R^{2}}\right) .
\end{aligned}
$$

The term $R\left(1+\frac{z}{R}\right)$ in (9) is a Lamé parameter. Eq. (9) is written in the traditional shell notation. This is obtained with the transformation $r=R+z$, which gives $\int_{R-h / 2}^{R+h / 2} r d r=\int_{-h / 2}^{h / 2} R(1+z / R) d z$.

3.3. Non-vanishing strains in case of incompressible Neo-Hookean material

For an incompressible Neo-Hookean material, the SED has the form [15]

$W_{N H I}=\frac{E}{6}\left(I_{1}-3\right)$,

where $I_{1}$ is the first invariant of the Cauchy-Green deformation tensor (3).

The expressions for the two strain components $\varepsilon_{x}$ and $\varepsilon_{z}$ have to be determined. To this end, the incompressibility condition $J=1$ [15] is enforced, where $J$ is given by expression (4).

From $L=h$, we also make the additional assumption that $\varepsilon_{z}=\varepsilon_{x}$ since (i) the cross-section of the ring is a square in this case, and (ii) the load is assumed to be just in direction $\theta$, so that the strains in the two orthogonal directions can be assumed to be the same, as for a beam of square section.

The incompressibility condition thus yields

$\varepsilon_{x}=\frac{1}{2 \sqrt{2 \varepsilon_{\theta}+1}}-\frac{1}{2}$,

and the SED becomes

$W_{N H I}=\frac{E}{3}\left(\frac{1}{\sqrt{2 \epsilon_{\theta}+1}}+\epsilon_{\theta}-1\right)$.

The expansion of $W_{N H I}$ into a series in $\varepsilon_{\theta}$ up to second power coincides with expression (8).

\subsection{Non-vanishing strains in case of compressible Neo-Hookean material in Ogden's form}

Two types of SED for compressible Neo-Hookean materials can be found in the literature. First, we consider Ogden's form given by [16]

$W_{N H O}=\frac{E}{4(1+v)}\left(I_{1}-3-2 \ln (J)\right)+\frac{E}{6(1-2 v)}(J-1)^{2}$.

The expressions of $\varepsilon_{x}$ and $\varepsilon_{z}$ in terms of $\varepsilon_{\theta}$, and thus $w$, are obtained by enforcing vanishing of the corresponding stresses. We impose the conditions of absence of the second Piola-Kirchhoff stresses, which are given by formulas $S_{x}=\frac{\partial W_{N H O}}{\partial \varepsilon_{x}}, S_{z}=\frac{\partial W_{N H O}}{\partial \varepsilon_{z}}$ [12]. Based on the relationships $\varepsilon_{x}=\left(\lambda_{x}^{2}-1\right) / 2$ and $\varepsilon_{z}=\left(\lambda_{z}^{2}-1\right) / 2$, the following equalities are obtained:

$\frac{\partial W_{N H O}}{\partial \lambda_{x}}=\frac{\partial W_{N H O}}{\partial \varepsilon_{x}} \frac{\partial \varepsilon_{X}}{\partial \lambda_{x}}=\frac{\partial W_{N H O}}{\partial \varepsilon_{x}} \lambda_{x}$

$\frac{\partial W_{N H O}}{\partial \lambda_{z}}=\frac{\partial W_{N H O}}{\partial \varepsilon_{z}} \frac{\partial \varepsilon_{z}}{\partial \lambda_{z}}=\frac{\partial W_{N H O}}{\partial \varepsilon_{z}} \lambda_{z}$

Therefore, we can satisfy the conditions $S_{x}=0, S_{z}=0$ equating $\frac{\partial W_{N H O}}{\partial \lambda x}, \frac{\partial W_{N H O}}{\partial \lambda_{z}}$ to zero. These conditions are equivalent since principal stretches are always positive [15].

$\frac{\partial W_{N H O}}{\partial \lambda_{X}}=\frac{E}{2(1+v)}\left(\lambda_{x}-\frac{1}{\lambda_{X}}\right)+\frac{E}{3(1-2 v)} \lambda_{\theta} \lambda_{z}(J-1)$,

$\frac{\partial W_{N H O}}{\partial \lambda_{z}}=\frac{E}{2(1+v)}\left(\lambda_{z}-\frac{1}{\lambda_{z}}\right)+\frac{E}{3(1-2 v)} \lambda_{x} \lambda_{\theta}(J-1)$.

Setting these two expressions to zero creates a system of two nonlinear algebraic equations in $\lambda_{x}, \lambda_{z}$, which can be solved analytically. This system has eight solutions, only one of which has a physical meaning:

$\lambda_{x}=\lambda_{z}=\frac{1}{\sqrt{2}}$

$\sqrt{\frac{-\frac{3(1-2 v)}{2(1+v)}+\lambda_{\theta}+\sqrt{\frac{6(1-2 v)}{(1+v)} \lambda_{\theta}^{2}+36(1-2 v)^{2}\left(\frac{1}{4(1+v)}-\frac{1}{6(1-2 v)} \lambda_{\theta}\right)^{2}}}{\lambda_{\theta}^{2}}}$.

To obtain the expression of the SED in terms of the deflection, expression (12) is substituted into Eq. (11) together with $\lambda_{\theta}=\sqrt{2 \varepsilon_{\theta}+1}$.

\subsection{Non-vanishing strains in case of compressible Neo-Hookean material in Bower's form}

The second form of the SED for compressible Neo-Hookean material can be found in the book by Bower [15]. The same form is used in the commercial finite element package ANSYS [17]. The expression for the SED in this case is 
$W_{N H B}=\frac{E}{4(1+v)}\left(I_{1} J^{-\frac{2}{3}}-3\right)+\frac{E}{6(1-2 v)}(J-1)^{2}$.

The conditions of absence of stresses are:

$\frac{\partial W_{N H B}}{\partial \lambda_{x}}=\frac{E}{2(1+v)} J^{-\frac{2}{3}}\left(\lambda_{x}-\frac{1}{3} \lambda_{\theta} \lambda_{z} I_{1} J^{-1}\right)+\frac{E}{3(1-2 v)} \lambda_{\theta} \lambda_{z}(J-1)=0$,

$\frac{\partial W_{N H B}}{\partial \lambda_{z}}=\frac{E}{2(1+v)} J^{-\frac{2}{3}}\left(\lambda_{z}-\frac{1}{3} \lambda_{x} \lambda_{\theta} I_{1} J^{-1}\right)+\frac{E}{3(1-2 v)} \lambda_{x} \lambda_{\theta}(J-1)=0$.

Analytical solutions of (14) are untrackable. Instead, the system can be solved numerically but an analytical expression for the SED in terms of $w$ is desired since this quantity has to be differentiated with respect to the generalized coordinate $w$ to derive the corresponding Lagrange equation. This is achieved by first solving the system (14) numerically, and then by approximating, through a least-squares technique, the solution as a polynomial in $w$.

\section{Governing Lagrange equation}

The behavior of the ring under pressure is properly captured by a single Lagrange equation:

$\frac{\partial U}{\partial w}=\frac{\partial F}{\partial w}$,

where $U=\int_{0}^{L} \int_{0}^{2 \pi} \int_{-\frac{h}{2}}^{\frac{h}{2}} W R\left(1+\frac{z}{R}\right) d x d \theta d z$ is the potential energy, $W$ is the SED, $w$ is the radial displacement, and $F$ is the virtual work done by pressure. Two pressure loads are investigated in this study. The first one is a displacement-independent pressure (uniformly distributed radial forces), which leads to

$F=\int_{0}^{L} \int_{0}^{2 \pi} P w R d \theta d x=2 \pi R L P w$,

where $P$ is the pressure magnitude. We assume that the pressure acts on the middle surface of the ring.

The second load is a displacement-dependent pressure (in the present case the area of application changes during the ring deformation and the force per unit area is kept constant). The expression of the area of the middle surface of the deformed ring is given by

$S_{d}=2 \pi(R+w) L\left(1+\varepsilon_{x}\right)$,

which can be normalized with respect to the area of the middle surface of the undeformed ring $S_{u}=2 \pi R L$ as follows:

$\frac{S_{d}}{S_{u}}=\left(1+\frac{w}{R}\right)\left(1+\varepsilon_{x}\right)$.

Finally, the work of the displacement-dependent pressure is $F=\int_{0}^{L} \int_{0}^{2 \pi} P w\left(1+\frac{w}{R}\right)\left(1+\epsilon_{x}\right) R d \theta d x=2 \pi R L P w\left(1+\frac{w}{R}\right)\left(1+\epsilon_{x}\right)$.

Eq. (15) can sometimes be solved analytically, even though numerical procedures are the only option in a vast majority of configurations.

\section{Numerical example}

As an example, we solve the problem for the ring depicted in Fig. 1 with the following parameters: $R=0.15 \mathrm{~m}, L=h=0.03 \mathrm{~m}, E=1.98 * 10^{11} \mathrm{~Pa}$; for the compressible material, $v=0.3$, while for the incompressible material, $v=0.5$.

\subsection{Linear material}

The equilibrium equation in this case is

$\frac{E L \pi h}{R}\left(2 w+\frac{3 w^{2}}{R}+\frac{w^{3}}{R^{2}}\right)=\frac{\partial F}{\partial w}$.

The two possible external forces are (i) the distributed radial forces

$\frac{\partial F}{\partial w}=2 \pi R L P$

and (ii) displacement-dependent pressure

$$
\begin{aligned}
& \frac{\partial F}{\partial w} \\
& =2 \pi R L P\left(\left(1+\frac{w}{R}\right)\left(1+\epsilon_{x}\right)+\frac{w}{R}\left(1+\epsilon_{x}\right)+w\left(1+\frac{w}{R}\right)\left(1+\frac{\partial \epsilon_{x}}{\partial w}\right)\right) \\
& =2 \pi R L P\left(\left(1+\frac{w}{R}\right)\left(1-v\left(\frac{w}{R}+\frac{w^{2}}{2 R^{2}}\right)\right)+\frac{w}{R}\left(1-v\left(\frac{w}{R}+\frac{w^{2}}{2 R^{2}}\right)\right)\right. \\
& \left.\quad+w\left(1+\frac{w}{R}\right)\left(1-v\left(\frac{1}{R}+\frac{w}{R^{2}}\right)\right)\right) .
\end{aligned}
$$

It is worthy to note that in the case of distributed radial forces, the equilibrium equation does not depend on Poisson's ratio.

The numerical results are plotted in Fig. 2(a) as solid lines and

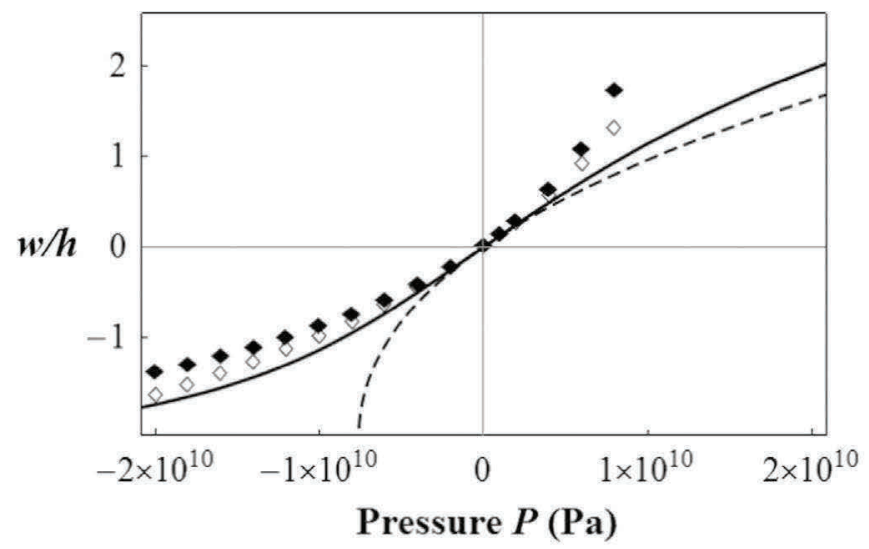

(a)

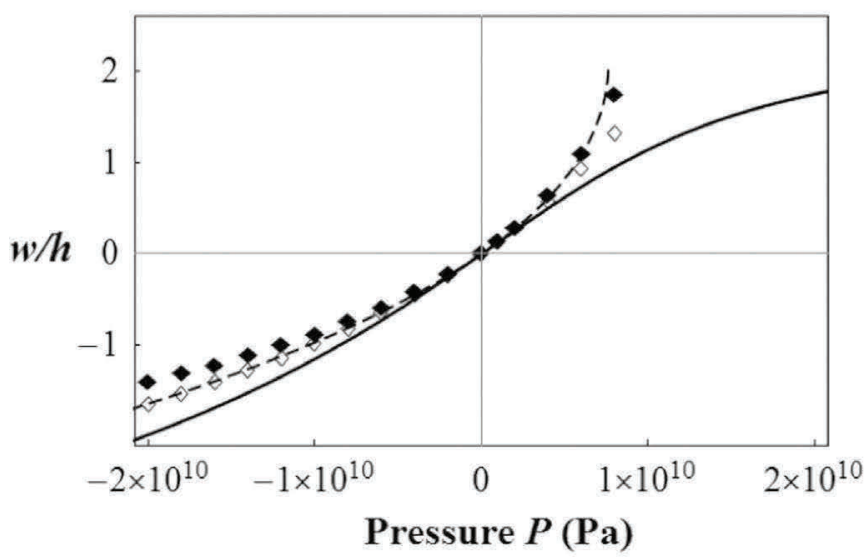

(b)

Fig. 2. Deflection of the ring for the linear material; solid line, displacement-dependent pressure; dashed line, distributed radial forces; $\bullet$, ANSYS results for ring under displacement-dependent pressure; $\diamond$, ANSYS results for ring under distributed radial forces. (a) actual results and (b) results in dashed and solid lines with reversed (opposite) signs of displacement and pressure (ANSYS results not reversed). 
show a large difference in case of distributed radial forces and displacement-dependent pressure. Finite element (FE) results obtained with ANSYS [17] are also displayed; they compare well only for small deflections, and are particularly far off in the case of distributed radial forces.

However, surprisingly, Fig. 2(b) shows that, switching between inward and outward directions, highly improves the agreement, and results become almost coincident for the case of distributed radial forces. It would be legitimate to suspect a sign error in our computations, but, as further investigations show (see the next subsection), this is not the case.

In order to verify our analytical solution, we used the FE code created by the research group of Professor Reddy [18]. The comparison of the results is given in Fig. 3 for distributed radial forces. These FE results are extremely close to our solution.

\subsection{Incompressible Neo-Hookean material}

For an incompressible Neo-Hookean material, the equilibrium equation is

$\frac{2 \pi E L h}{3}\left(1+\frac{w}{R}-\frac{1+\frac{w}{R}}{\left(2 \varepsilon_{\theta}+1\right)^{\frac{3}{2}}}\right)=\frac{\partial F}{\partial w}$.

The term $\frac{\partial F}{\partial w}$, in case of distributed radial forces, has the form (16); and in case of displacement-dependent pressure, it is:

$$
\begin{aligned}
\frac{\partial F}{\partial W}= & 2 \pi R L P\left(\left(1+\frac{w}{R}\right)\left(1+\varepsilon_{X}\right)+\frac{w}{R}\left(1+\varepsilon_{\chi}\right)+w\left(1+\frac{w}{R}\right)\right. \\
& \left.\left(1-\frac{\frac{1}{R}+\frac{w}{R^{2}}}{2\left(2 \varepsilon_{\theta}+1\right)^{\frac{3}{2}}}\right)\right) .
\end{aligned}
$$

The two strains $\varepsilon_{\theta}$ and $\varepsilon_{\chi}$, arising in expressions (17) and (18), are defined in (5) and (10), respectively.

Eq. (17) is solved numerically and results are presented in Fig. 4. In this case, ANSYS results are particularly close (coincident in case of distributed radial forces for the full pressure range) to our results without changing the sign. Also, the difference in the radial displacement $w$ between distributed radial forces and actual (displacement-dependent) pressure is not that large, except for very large pressures.

Since the results for the ring made of linear material subjected to distributed radial forces do not depend on Poisson's ratio, they can easily be compared to the results for the incompressible Neo-

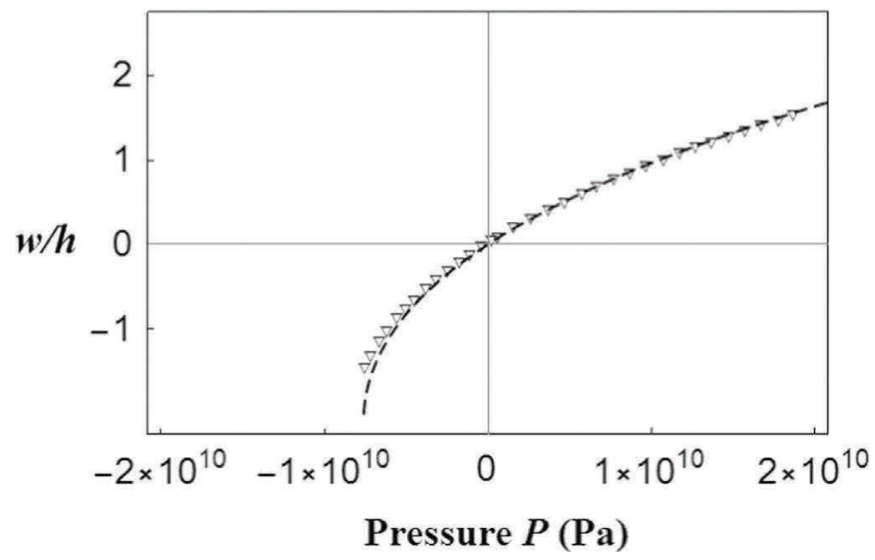

Fig. 3. Deflection of the ring for the linear material; dashed line, distributed radial forces; $\nabla$, results from FE code by Reddy [18] for ring under distributed radial forces.

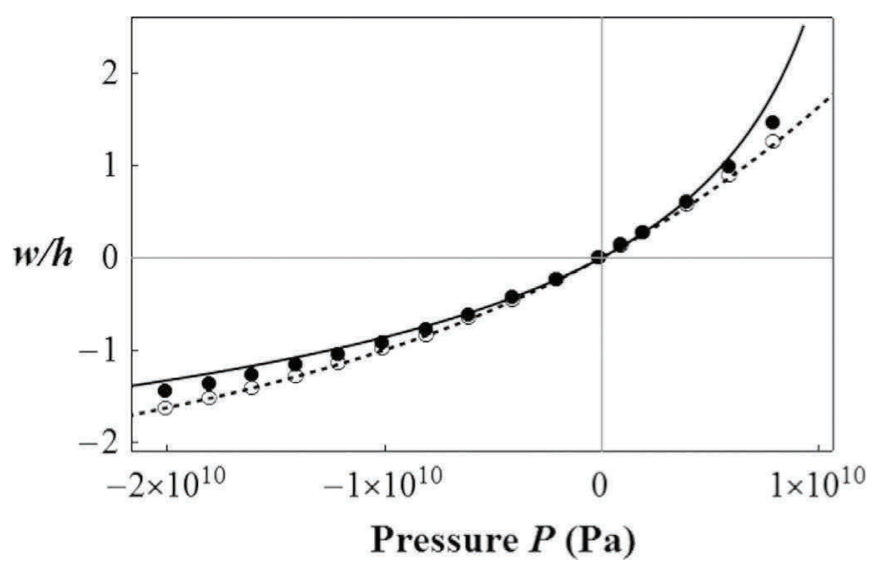

Fig. 4. Deflection of the ring for the incompressible Neo-Hookean ring; solid line, displacement-dependent pressure; dashed line, distributed radial forces; $\bullet$, ANSYS results for ring under displacement-dependent pressure $(\nu=0.5)$; $(\circ)$, ANSYS results for ring under distributed radial forces $(\nu=0.5)$.

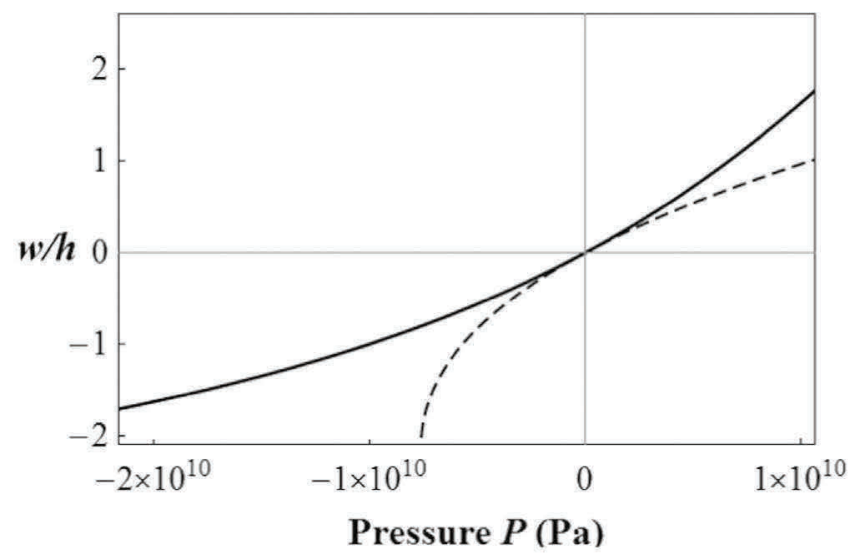

(a)

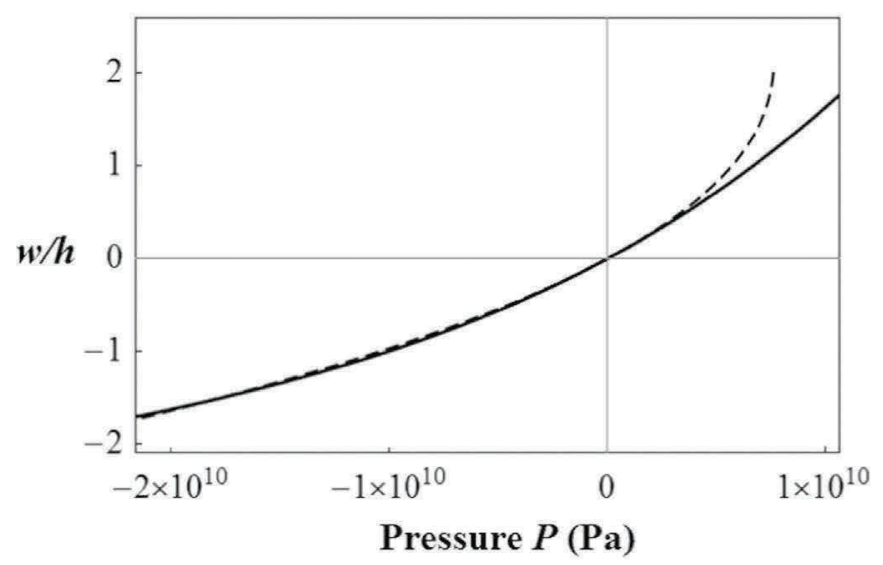

(b)

Fig. 5. Deflection of the ring for incompressible Neo-Hookean (solid line) and linear material (dashed line) materials and subjected to distributed radial forces. (a) Actual results; (b) Results for linear material plotted with opposite signs (exchanging inward and outward directions).

Hookean material, as shown in Fig. 5(a). Again, a good match is observed in the compression and extension zones only for a very small pressure range. However, for the linear material, if the inward and outward directions are exchanged (see Fig. 5(b)), the match becomes surprisingly good for a wide pressure range. This suggests that, rings of Neo-Hookean and linear materials behave in an opposite way, unlike rods under uniaxial tension made of these 
materials [12].

To understand such a strange and unexpected feature, the SED of the Neo-Hookean material is expanded into a series in the deflection $w$ :

$W_{N H I}=E\left(\frac{w^{2}}{2 R^{2}}-\frac{w^{3}}{3 R^{3}}+\frac{w^{4}}{3 R^{4}}+\cdots\right)$

On the other hand, the SED of the linear material is (8) and (9)

$W_{L}=E\left(\frac{w^{2}}{2 R^{2}}+\frac{w^{3}}{2 R^{3}}+\frac{w^{4}}{8 R^{4}}\right)$.

We can see, comparing Eqs. (20) and (21), that the cubic terms have opposite signs; this explains the almost mirrored behavior of the two rings. Both compressible Neo-Hookean materials also exhibit a cubic term with a minus sign in the SED series expansion, and their series expansions tend to series (20) as $v$ approaches 0.5. These series are not shown here for the sake of conciseness.

\subsection{Compressible Neo-Hookean materials}

For the two formulations of compressible Neo-Hookean material, the equilibrium equations can be obtained as described in Section 4: they are lengthy and are not provided here. For a compressible NeoHookean material in Bower's form, the equation is not exact, since the system $S_{x}=0, S_{z}=0$ for $\varepsilon_{x}, \varepsilon_{z}$ is solved numerically and subsequently approximated by a polynomial in $w$. The strains $\varepsilon_{x}$ and $\varepsilon_{z}$ are identical in our problem. A convergence analysis shows that a sixth-order polynomial in $w$ is sufficient for good accuracy. This is illustrated in Fig. 6, which displays the comparison between numerical results and polynomial approximation; the maximal relative error is less than $0.1 \%$. This justifies the choice of the polynomial expression in the following calculations.

The pressure-radial deformation curves for Neo-Hookean materials are shown in Figs. 7 and 8, for distributed radial forces and displacement-dependent pressure, respectively. For the compressible NeoHookean material, Bower's model is supposedly used in the ANSYS code [17]. In case of distributed radial forces (Fig. 7), FE results for $\nu=0.3$ are close to our results for Bower's model, but also to the results for incompressible material. In case of displacement-dependent pressure (Fig. 8), FE results for $\nu=0.3$ are closer to incompressible and Ogden's compressible Neo-Hookean materials than to Bower's material. Generally, FE results for distributed radial forces are extremely close to our results, while this agreement is slightly reduced for the case of displacement-dependent pressure when compared to Bower's compressible model.

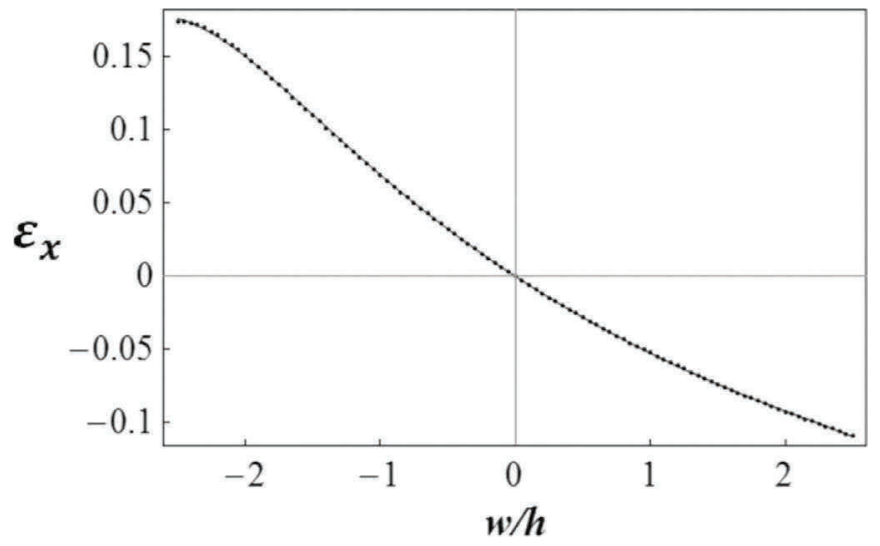

Fig. 6. Strain $\varepsilon_{X}$ found numerically (dots) and the sixth-order polynomial approximation (solid line).

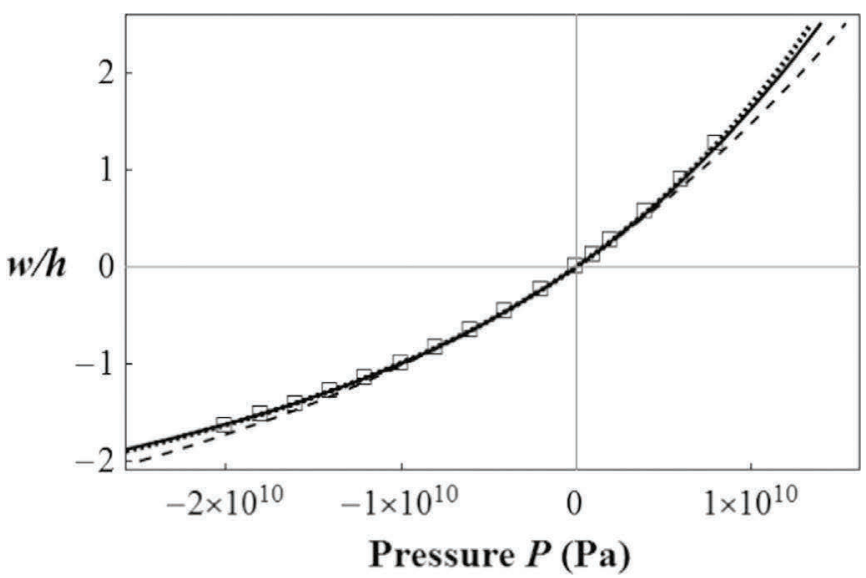

Fig. 7. Deflection of the Neo-Hookean rings subjected to distributed radial forces Solid line, incompressible material; dotted line, Bower's compressible material $(\nu=0.3)$; dashed line, Ogden's compressible material $(\nu=0.3)$; $\square$, ANSYS results for compressible Neo-Hookean material $(\nu=0.3)$.

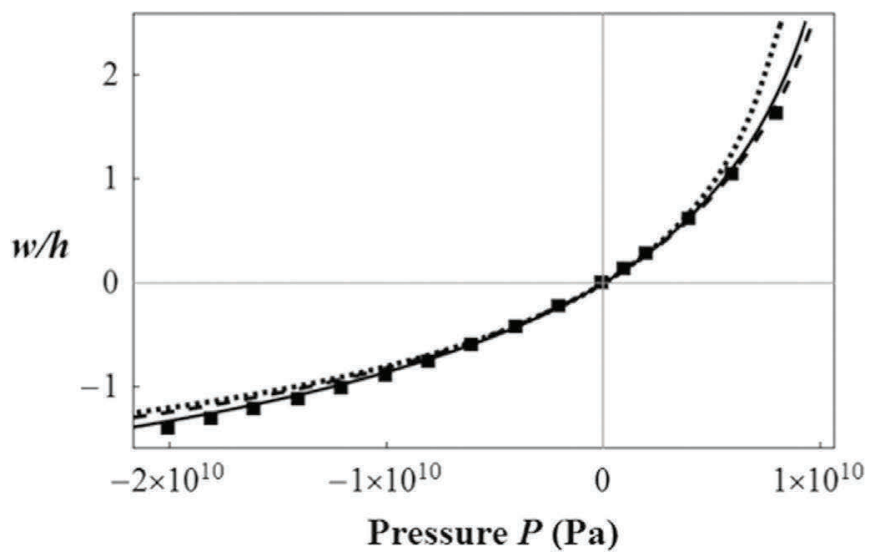

Fig. 8. Deflection of the Neo-Hookean rings subjected to displacement-dependent pressure. Solid line, incompressible material; dotted line, Bower's compressible material $(\nu=0.3$ ); dashed line, Ogden's compressible material $(\nu=0.3)$; $\bullet$, ANSYS results for compressible Neo-Hookean material $(\nu=0.3)$.

\section{Discussion and conclusion}

We have analyzed the static, axially symmetric, deflection of a ring under internal and external pressure. Compressible and incompressible Neo-Hookean materials, as well as linear elastic material, are implemented. Results obtained from the theory developed in Section 5 are systematically compared to those computed by the finite element package ANSYS. Since we found that ANSYS is giving inaccurate results in case of linear elastic material at large strains, we also repeated this analysis by using the commercial FE program ABAQUS. The results obtained by both finite element codes are practically identical. Instead, a non-commercial FE code developed by the research group of Professor Reddy [18] (this code has been developed only for linear elastic materials, so it does not use the invariants of the Cauchy-Green deformation tensor in order to evaluate the strain energy of the structure) has given results almost coincident to our analytical results, giving strength to our solution and confirming that commercial FE codes, at least ANSYS and ABAQUS, give incorrect results at large strains when a linear elastic material is selected for the analysis. This is a quite surprising result.

Our two main conclusions are the following:

- As opposed to the simple uniaxial tension of a rod, results for rings of linear and Neo-Hookean materials greatly differ and 
surprisingly exhibit opposite behaviors. The cubic term in the governing Lagrange equation has opposite sign, which leads to almost mirrored behavior. The pressure-deflection curves for a Neo-Hookean ring under external distributed radial forces, and for a ring of linear material under internal distributed radial forces, coincide up to high deflections (2/5 of radius in our example). Correspondence for the second pair of curves, those for displacement-dependent pressure, is not that good; however, the pressure-deflection curves are close for the deflection up to $1 / 10$ of radius.

- Finite element results for the Neo-Hookean materials are in agreement to the results proposed in this work. The agreement is particularly good for a radial force while this agreement is slightly reduced for a displacement-dependent pressure. However, within ANSYS, linear and Neo-Hookean materials behave similarly. This suggests that the "linear elastic isotropic" material available in ANSYS (and ABAQUS) is actually not linearly elastic. In fact, for large strains, it exhibits a behavior that is much closer to a hyperelastic material than to a linear elastic material.

\section{Acknowledgments}

The authors acknowledge the financial support of the NSERC Discovery Grant, Canada Research Chairs and the Qatar National Research Fund NPRP 7-032-2-016.

\section{References}

[1] G. Lame, Leçons sur la théorie mathématique de l'élasticité des corps solides, Bachelier, Paris, 1852

[2] A.E.H. Love, A Treatise on the Mathematical Theory of Elasticity, 4th edition, Cambridge University Press, Cambridge, 1927.

[3] A.I. Lurie, Theory of Elasticity, Springer-Verlag, Berlin, Heidelberg, 2005.

[4] D.M. Haughton, R.W. Ogden, Bifurcation of inflated circular cylinders of elastic material under axial loading-II. Exact theory for thick-walled tubes, J. Mech. Phys. Solids 27 (1979) 489-512.

[5] Y. Zhu, X.Y. Luo, R.W. Ogden, Asymmetric bifurcations of thick-walled circular cylindrical elastic tubes under axial loading and external pressure, Int. J. Solids Struct. 45 (2008) 3410-3429.

[6] Y. Zhu, X.Y. Luo, R.W. Ogden, Nonlinear axisymmetric deformations of an elastic tube under external pressure, Eur. J. Mech. A/Solids 29 (2010) 216-229.

[7] Y. Zhu, X.Y. Luo, H.M. Wang, R.W. Ogden, C. Berry, Three-dimensional nonlinear buckling of thick-walled elastic tubes under pressure, Int. J. Non-linear Mech. 48 (2013) 1-14.

[8] H.A. Erbay, H. Demiray, Finite axisymmetric deformations of elastic tubes: an approximate method, J. Eng. Math. 29 (1995) 451-472.

[9] Y.C. Chen, D.M. Haughton, Stability and bifurcation of inflation of elastic cylinders, Proc. R. Soc. Lond. A 459 (2003) 137-156.

[10] M. Heil, T.J. Pedley, Large axisymmetric deformation of a cylindrical shell conveying a viscous flow, J. Fluids Struct. 9 (1995) 237-256.

[11] P. Kozlovsky, U. Zaretsky, A.J. Jaffa, D. Elad, General tube law for collapsible thin and thick-wall tubes, J. Biomech. 47 (2014) 2378-2384.

[12] I. Breslavsky, M. Amabili, M. Legrand, Physically and geometrically non-linear vibrations of thin rectangular plates, Int. J. Non-linear Mech. 58 (2014) 30-40.

[13] I.D. Breslavsky, M. Amabili, M. Legrand, Nonlinear vibrations of thin hyperelastic plates, J. Sound Vib. 333 (2014) 4668-4681.

[14] M. Amabili, Nonlinear Vibrations and Stability of Shells and Plates, Cambridge University Press, New York, 2008.

[15] A. Bower, Applied Mechanics of Solids, CRC Press, Boca Raton, Taylor and Francis Group, 2010.

[16] R. Ogden, Non-linear Elastic Deformations, Dover Publications, New York, 1997.

[17] H.H. Lee, Finite Element Simulations with ANSYS Workbench 14. Mission, SDC Publications, 2012

[18] G.S. Payette, J.N. Reddy, A seven-parameter spectral/hp finite element formulation for isotropic, laminated composite and functionally graded shell structures, Comp Methods Appl Mech Eng 278 (2014) 664-704. 